sich in der Regel auf ausländische Staatsbürger. In welchem Ausmaß sich der Wohnkontext der „Neoösterreicher“ verändert, war bislang wenig bekannt. Die wichtige Frage der Wohnintegration von Zuwanderern möchte der nun vorliegende Forschungsbericht beantworten. Er widmet sich auf der theoretischen Grundlage der klassischen Sozialökologie den sozialräumlichen Milieus im Wiener Stadtraum und vergleicht auf der Basis der Daten der amtlichen Statistik die Wohngebiete der nichteingebürgerten mit jenen der eingebürgerten Migranten. Den theoretischen Prämissen der Sozialökologie gemäß müssten „erfolgreiche“ Migranten (d.h. Eingebürgerte) die sozialräumlichen Milieus ihrer ,ethnic communities“ verlassen und sich in die Wiener Bevölkerung ,untermischen“.

\title{
2 Forschungsfrage und Datenbasis
}

Die Basis dieser Studie bildet ein Vergleich zwischen ausländischen Staatsbürgern und „Neoösterreichern“. Die empirische Grundlage setzt sich aus Daten der amtlichen Statistik sowie selbst akquirierten qualitativen Daten zusammen. Die räumliche Bezugsebene ist die Bundeshauptstadt Wien. Die folgenden Fragen sollen beantwortet werden:

- Welche Auswirkungen besitzen die Annahme der österreichischen Staatsbürgerschaft sowie die Aufenthaltsdauer auf die Wohnsituation und die Wohnintegration von Zuwanderern in Wien?

- Welche Prozesse vollziehen sich im Hinblick auf die ethnische Segregation? Löst sich diese mit der Einbürgerung auf, wie es den theoretischen Prämissen der Sozialökologie entspräche, oder sind residentielle Segregationsmuster stabil?

- Ist der Staatsbürgerschaftserwerb in Bezug auf die Wohnintegration als ein Indiz für eine ,gelungene Integration“ zu bewerten, d.h., manifestiert sich darin der letzte Schritt eines „erfolgreichen“ Migrationspfades? Sind also jene Migranten, die sich einbürgern lassen, auch die integrierten mit guten Wohnverhältnissen und mit einem sozioökonomisch höheren Status?

- Lassen sich herkunftsgruppenspezifische Unterschiede feststellen?

Die beiden Analyseebenen des Projektes sind also:

- die Wohnintegration von ausländischen Staatsbürgern und Neoösterreichern auf dem Wiener Wohnungsmarkt und

- die sozialräumlichen Strukturen im Wiener Stadtraum: Die Basis bildet eine Analyse der räumlichen Verteilung der Wohnstandorte der eingebürgerten versus jene der nichteingebürgerten Migranten.

Die beiden wichtigsten amtlichen Datenquellen, die einer Verkettung unterzogen wurden, waren

(1) die Volkszählung 2001 sowie die

(2) Häuser- und Wohnungszählung 2001. 
Die Volkszählung beinhaltete erstmals im Jahr 2001 die Frage nach dem Geburtsland des Zensiten. Die neue Variable „Geburtsland“ und die traditionelle Variable „Staatsbürgerschaft“ ermöglichten auf die Gruppe der Eingebürgerten bezogene Analysen. Die ISIS-Datenbank deckt die nötigen Daten auf den entsprechenden räumlichen Aggregationsebenen in auswertbarer Form ab, daher erfolgte der Ankauf entsprechender Sonderauswertungen bei Statistik Austria auf der räumlichen Bezugsebene der Zählsprengel $(\mathrm{n}=1.400)$. Diese Daten bildeten die Grundlage für die Erstellung eines Sets von Verteilungskarten mit ArcViewGIS, welche die Prozesse der räumlichen Segregration visualisieren.

Die Daten der amtlichen Statistik vermögen zwar eine erhebliche Bandbreite an Fragen bezüglich der Wohnintegration zu beantworten, vermitteln jedoch einen eingeschränkten Zugang zur Thematik. Daher wurde zusätzlich ein qualitativer Zugang gewählt und 11 narrative Interviews und ein Experteninterview durchgeführt. Die Interviews sollten Aufschlüsse über das Wie und Warum der Einbürgerung, die daran geknüpften individuellen Erwartungen sowie die daraus resultierenden Konsequenzen für die Wohnsituation der Migranten vermitteln.

\section{$3 \quad$ Einbürgerung und Integrationsprozesse}

In allen europäischen Zuwanderungsländern werden Diskussionen um die Integration der Migrantenbevölkerungen geführt. Der Großteil der Analysen zur Einbürgerung fokussiert auf den Einfluss der Übernahme der Staatsangehörigkeit auf die Integration im Aufnahmeland: „Naturalization may be the ultimate step in migration, as it represents attachment to country of destination, even including, for nationals of countries who do not allow dual citizenship, renouncing to former citizenship. As such, it may also seem to be a major aspect of integration, with adoption of social and political values linked with the constitution of the host country " (Rallu 2004: 1). Viele Studien betonen die Wichtigkeit sozioökonomischer, kultureller und demographischer Variablen. Die Frage nach der Richtung des Kausalzusammenhanges zwischen Integration und Einbürgerung konnte bislang nicht schlüssig beanwortet werden: „In fact, it is obviously impossible to know if naturalization is an effect of successful integration, and of integration efforts done by immigrants who want to obtain citizenship, or if it is a cause that leads to better integration for some groups of the immigrant population " (Fougère \& Safi 2006). Die Übernahme der Staatsbürgerschaft des Migrationsziellandes wird in der öffentlichen Meinung häufig unhinterfragt als letzter Schritt des Integrationsprozesses aufgefasst. Hierbei stellt sich jedoch die Frage, ob es sich nicht vielmehr doch nur um einen - zwar wichtigen - Zwischenschritt handelt, der die Integration und die Bindung an das Zielland verstärkt, primär bezogen jedoch auf den soziökonomischen Aspekt der Integration.

Die Einbürgerung - dies wurde empirisch vielfach nachgewiesen - wirkt sich in erster Linie auf die Arbeitsmarktpositionierung der Zuwanderer und deren Chancen, 